\title{
ISOTROPIC-POLYHARMONIC B-SPLINES AND WAVELETS
}

\author{
Dimitri Van De Ville, Thierry Blu, Brigitte Forster, Michael Unser \\ Biomedical Imaging Group, STI/IOA \\ Swiss Federal Institute of Technology Lausanne (EPFL) \\ CH-1015 Lausanne EPFL, Switzerland
}

\begin{abstract}
We propose the use of polyharmonic B-splines to build non-separable two-dimensional wavelet bases. The central idea is to base our design on the isotropic polyharmonic B-splines, a new type of polyharmonic B-splines that do converge to a Gaussian as the order increases. We opt for the quincunx subsampling scheme which allows us to characterize the wavelet spaces with a single wavelet: the isotropic-polyharmonic B-spline wavelet. Interestingly, this wavelet converges to a combination of four Gabor atoms, which are well separated in frequency domain. We also briefly discuss our Fourier-based implementation and present some experimental results.
\end{abstract}

\section{INTRODUCTION}

Multiresolution analysis and wavelets have proven to be a very useful framework for image processing. The fundamental theory of the discrete wavelet transform can be formulated in the continuous domain using embedded signal spaces spanned by scaling functions, and their orthogonal complements spanned by wavelets. These functions are related to each other via digital filters for Mallat's fast wavelet transform algorithm.

Most two-dimensional wavelet transforms use tensor products of one-dimensional basis functions. Unfortunately, these tend to favor horizontal and vertical directions; they also create a "diagonal" cross-term that does not have a straightforward interpretation. We distinguish between two viable options for the design of better non-separable wavelet transforms: directionality and isotropy. In the first case, one pursues maximal angular selectivity. The second option of emphasizing isotropy is interesting for image processing as well, but it has received considerably less attention. A strong motivating factor is that many standard image processing algorithms exploit the rotation-invariant properties of filters such as the Gaussian and Laplacian.

In this paper, we introduce new non-separable wavelet bases. Our construction starts from isotropic polyharmonic B-splines, which are improved versions of Rabut's elementary polyharmonic B-splines. Unlike for Rabut's, we can prove that these new B-splines are guaranteed to converge to a Gaussian as their order increases, in contrast with their elementary counterparts. Next, we define a multi-resolution analysis based on these scaling functions using the quincunx subsampling scheme. We derive the corresponding family of semi-orthogonal wavelets focussing on one that is particularly interesting: the isotropic-polyharmonic B-spline wavelet. This wavelet too is particularly well localized in space and frequency because it converges to a combination of Gabor atoms (occupying the corners of the Nyquist region) as the order increases. Finally, we briefly mention the implementation in the Fourier domain and we show some results that are relevant to image processing.

\section{POLYHARMONIC B-SPLINES}

\subsection{Elementary polyharmonic B-splines}

The "elementary $\gamma / 2$-harmonic B-splines" where first introduced by Rabut [1]. In the Fourier domain, their definition is remarkably similar to the univariate B-spline case:

$$
\begin{aligned}
\phi_{\gamma}(\mathbf{x}) \leftrightarrow \hat{\phi}_{\gamma}(\boldsymbol{\omega}) & =\frac{\|2 \sin (\boldsymbol{\omega} / 2)\|^{\gamma}}{\|\boldsymbol{\omega}\|^{\gamma}} \\
& =\left(\frac{4 \sin ^{2}\left(\omega_{1} / 2\right)+4 \sin ^{2}\left(\omega_{2} / 2\right)}{\omega_{1}^{2}+\omega_{2}^{2}}\right)^{\frac{\gamma}{2}}
\end{aligned}
$$

with $\sin \omega=\left(\sin \omega_{1}, \sin \omega_{2}\right)$. The parameter $\gamma$ is the order, with $\gamma \in \mathbb{R}$ and $\gamma>1$. The elementary polyharmonic B-spline can be regarded as the convolution of a localization operator (the numerator in the Fourier domain expression), which is the $\gamma / 2$-th iterate of the most elementary discretization of the Laplacian operator, with a radial basis function (RBF) of degree $\gamma-2$.

The elementary polyharmonic B-splines satisfy many interesting properties; e.g., Riesz basis generation, convolution relation $\phi_{\gamma_{1}+\gamma_{2}}=\phi_{\gamma_{1}} * \phi_{\gamma_{2}}$, partition of unity, tunable order of approximation $\gamma$, total positivity $\left(\hat{\phi}_{\gamma}>0\right)$. It has been shown before [2] that the elementary polyharmonic Bsplines are perfectly valid scaling functions for a whole variety of scaling relations. However, and despite the fact that they are generated by multiple convolutions, we have observed that they fail to converge to a Gaussian when the order increases, as one would normally expect. The reason for this is that the central limit theorem is not applicable since they do not have a well-defined second-order moment. This means that they do not decrease fastly enough and that they are never optimally space-frequency-localized. Moreover, they exhibit priviledged directions as shown in Fig. 1 (a); e.g., consider $\omega_{1} \pm \omega_{2}=0$. 
(a)

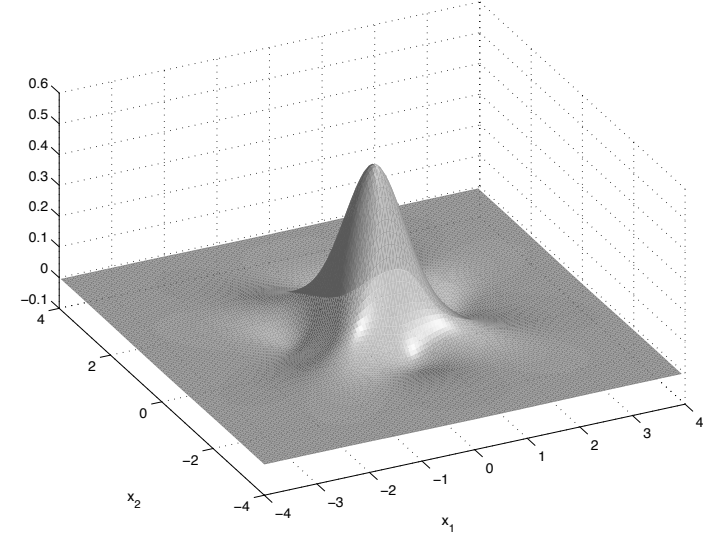

(b)

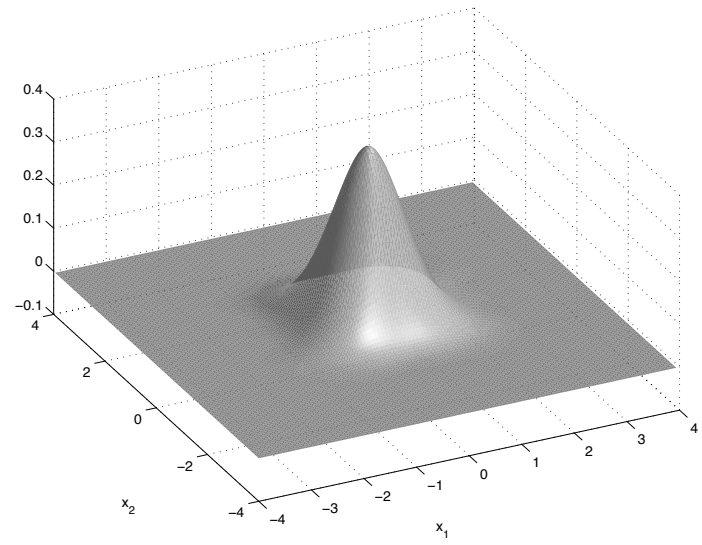

Fig. 1. Polyharmonic B-splines of order $\gamma=5$. (a) The elementary polyharmonic B-spline $\phi_{5}$. (b) The isotropic polyharmonic B-spline $\beta_{5}$.

\subsection{Isotropic polyharmonic B-splines}

Here, we propose to improve the properties of the elementary polyharmonic B-splines by introducing a more isotropic version of the localization filter, which is a discretization of an iterated Laplacian. Our aim is to change the localization filter in the slightest way, such that the secondorder moment becomes well-defined and the Gaussian convergence is guaranteed. So we introduce the isotropic polyharmonic B-splines of order $\gamma$ in the Fourier domain as follows

$$
\begin{aligned}
\hat{\beta}_{\gamma}(\boldsymbol{\omega})= & \left(\frac{4 \sin ^{2}\left(\omega_{1} / 2\right)+4 \sin ^{2}\left(\omega_{2} / 2\right)}{\omega_{1}^{2}+\omega_{2}^{2}}\right. \\
& \left.-\frac{8}{3} \frac{\sin ^{2}\left(\omega_{1} / 2\right) \sin ^{2}\left(\omega_{2} / 2\right)}{\omega_{1}^{2}+\omega_{2}^{2}}\right)^{\frac{\gamma}{2}},
\end{aligned}
$$

which has a Taylor series development $\hat{\beta}_{\gamma}(\boldsymbol{\omega})=1-$ $\gamma\|\boldsymbol{\omega}\|^{2} / 24+O\left(\|\boldsymbol{\omega}\|^{4}\right)$, and as such a well-defined (isotropic) second-order moment. We can show that this isotropic version of the polyharmonic B-splines spatially decays like $1 /\|\mathbf{x}\|^{6}$, versus $1 /\|\mathbf{x}\|^{4}$ for its elementary counterpart (for $\gamma \geq 2$ ).

For a more detailed exposition of the isotropic polyharmonic B-splines and their properties in $N$ dimensions, we refer to our paper [3].

\section{MULTI-RESOLUTION ANALYSIS WITH QUINCUNX SUBSAMPLING}

We define the dilation matrix $\mathbf{D}$ that maps $\mathbf{k} \in \mathbb{Z}^{2}$ to $\mathbf{D k}$, which is a subset of $\mathbb{Z}^{2}$. The signal space spanned by the isotropic polyharmonic B-spline at a resolution $\mathbf{D}^{i}$ is

$$
\mathcal{V}_{\mathbf{D}^{i}}=\mathrm{cl}_{L^{2}\left(\mathbb{R}^{2}\right)} \operatorname{span}_{\mathbf{n} \in \mathbb{Z}^{2}}\left\{\beta_{\gamma}\left(\mathbf{D}^{i} \mathbf{x}-\mathbf{n}\right): n \in \mathbb{Z}^{2}\right\} .
$$

From now on, we select the quincunx subsampling scheme, which provides a slower progression through scales than the dyadic one $[4,5]$. The subsampling rate at each iteration is 2 such that a single wavelet spans the wavelet space at each resolution. The dilation matrix is given by

$$
\mathbf{D}=\left[\begin{array}{cc}
1 & 1 \\
1 & -1
\end{array}\right]
$$

The scaling filter associated with the isotropic polyharmonic B-spline enables us to map $\beta_{\gamma}$ to a coarser resolution as $\beta_{\gamma}(\mathbf{x})=\sum_{\mathbf{n}} b_{\gamma}(\mathbf{n}) \beta_{\gamma}(\mathbf{D x}-\mathbf{n})$. It can be derived in the Fourier domain as

$$
\begin{aligned}
& B_{\gamma}\left(e^{j \boldsymbol{\omega}}\right)=|\operatorname{det}(\mathbf{D})| \frac{\hat{\beta}_{\gamma}\left(\mathbf{D}^{\mathrm{T}} \boldsymbol{\omega}\right)}{\hat{\beta}_{\gamma}(\boldsymbol{\omega})}=2^{1-\frac{\gamma}{2}} . \\
& \quad\left(\frac{\sin ^{2}\left(\frac{\omega_{1}+\omega_{2}}{2}\right)+\sin ^{2}\left(\frac{\omega_{1}-\omega_{2}}{2}\right)-\frac{2}{3} \sin ^{2}\left(\frac{\omega_{1}+\omega_{2}}{2}\right) \sin ^{2}\left(\frac{\omega_{1}-\omega_{2}}{2}\right)}{\sin ^{2}\left(\frac{\omega_{1}}{2}\right)+\sin ^{2}\left(\frac{\omega_{2}}{2}\right)-\frac{2}{3} \sin ^{2}\left(\frac{\omega_{1}}{2}\right) \sin ^{2}\left(\frac{\omega_{2}}{2}\right)}\right)^{\frac{\gamma}{2}} .
\end{aligned}
$$

\section{ISOTROPIC-POLYHARMONIC B-SPLINE WAVELETS}

In this paper, we consider the semi-orthogonal design procedure as in [6]: at each scale, the two signal spaces spanned by the scaling function and the wavelet are orthogonal.

The wavelet $\psi\left(\mathbf{D}^{-1} \mathbf{x}\right)=\sum_{\mathbf{n}} w(\mathbf{n}) \beta_{\gamma}(\mathbf{x}-\mathbf{n})$ and its translates span the space $\mathcal{W}_{\mathbf{D}^{1}}$, which is included in the space $\mathcal{V}_{\mathbf{D}^{0}}=\mathcal{V}_{\mathbf{D}^{1}} \oplus \mathcal{W}_{\mathbf{D}^{1}}$ at a finer scale. The orthogonality condition between the wavelet and the scaling function at scale $\mathbf{D}^{-1}$ leads to the following condition in the $z$-domain:

$$
W(\mathbf{z}) B(\mathbf{z}) A(\mathbf{z})+W(-\mathbf{z}) B(-\mathbf{z}) A(-\mathbf{z})=0,
$$

where $A\left(e^{j \boldsymbol{\omega}}\right)=\sum_{\mathbf{n}}\left|\hat{\beta}_{\gamma}(\boldsymbol{\omega}+2 \pi \mathbf{n})\right|^{2}$ represents the autocorrelation filter of $\beta_{\gamma}$. By substitution, we see that the 
(a)

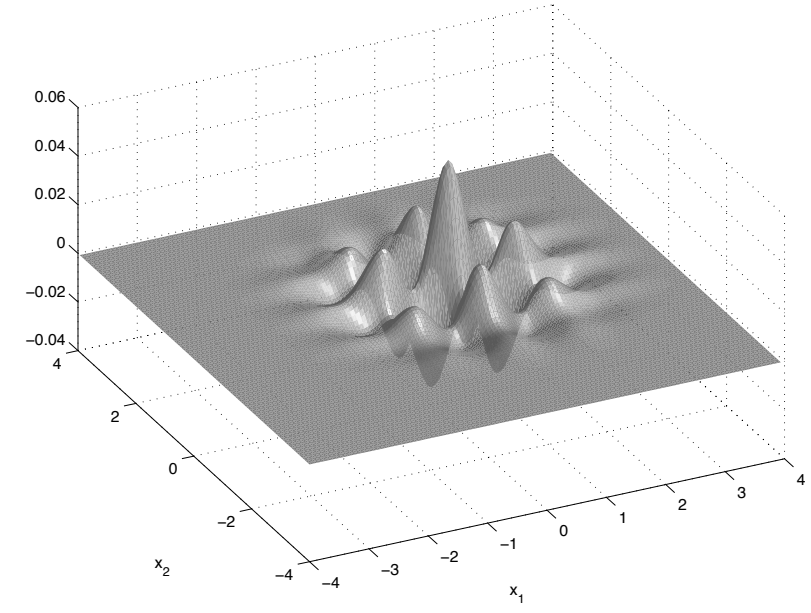

(b)

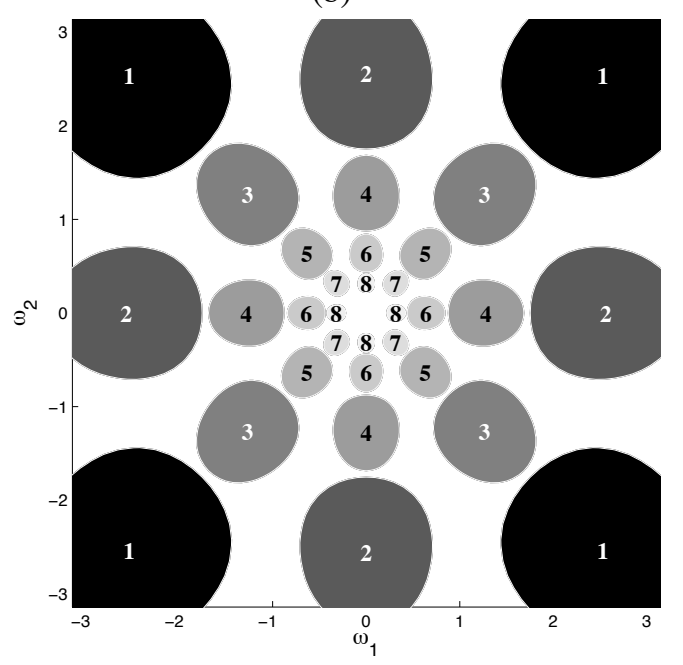

(c)

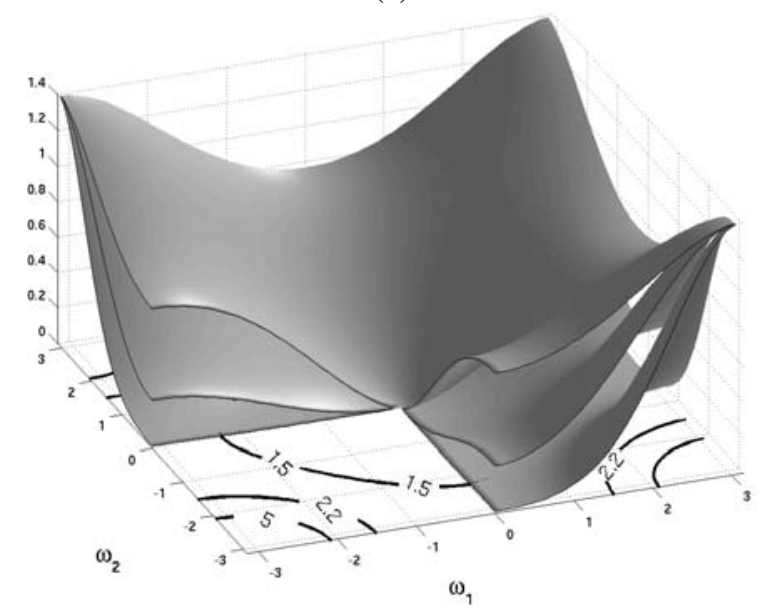

Fig. 2. (a) Isotropic-polyharmonic B-spline wavelet $\psi$ of order $\gamma=5$. (b) Tiling of the frequency domain for this wavelet and eight consecutive iterations. (c) Frequency response of the wavelet filter $W\left(e^{j \omega}\right)$ for orders $\gamma=1.5,2.2,5$. The contour lines correspond to $50 \%$ levels. general solution of this equation is

$$
W(\mathbf{z})=-z_{1}^{-1} Q\left(\mathbf{z}^{\mathbf{D}}\right) B_{\gamma}\left(-\mathbf{z}^{-1}\right) A(-\mathbf{z})
$$

where $Q(\mathbf{z})$ is a polynomial in $\mathbf{z}$ and $\mathbf{z}^{\mathbf{D}}$ is a shortcut notation for $\left(z_{1} z_{2}, z_{1} z_{2}^{-1}\right)$. The most obvious choice ${ }^{1}$ is $Q(\mathbf{z})=1$, which gives

$$
\hat{\psi}\left(\mathbf{D}^{\mathrm{T}} \boldsymbol{\omega}\right)=\frac{W\left(e^{j \boldsymbol{\omega}}\right)}{2} \hat{\beta}_{\gamma}(\boldsymbol{\omega}),
$$

with $W\left(e^{j \boldsymbol{\omega}}\right)=-e^{-j \omega_{1}} B_{\gamma}\left(e^{-j(\boldsymbol{\omega}+\boldsymbol{\pi})}\right) A\left(e^{j(\boldsymbol{\omega}+\boldsymbol{\pi})}\right)$, where $\pi=(\pi, \pi)$. This wavelet is referred to as the "isotropicpolyharmonic B-spline wavelet”.

The isotropic-polyharmonic B-spline wavelets (for an example, see Fig. 2 (a)) have several important properties.

1. Their shifted versions form a Riesz basis of the wavelet space at each resolution scale.

2. They behave as a $\gamma / 2$-th iterate of the isotropic Laplacian operator for low frequencies; i.e., $\hat{\psi}(\boldsymbol{\omega}) \propto\|\boldsymbol{\omega}\|^{\gamma}$.

3. They converge to the sum of four Gabor atoms, well separated in frequency domain, as the order $\gamma$ increases (for the proof, see [3]).

In Fig. 2 (b) we show their tiling in the frequency domain for eight consecutive iterations. Wavelet-like Gabor decompositions have already been successfully applied to many image processing tasks; e.g., edge detection, texture analysis, vision modelling. In Fig. 2 (c) we show the frequency response of the wavelet filter. For low order, the filter is isotropic for quite a large region in the Nyquist region. In this "Laplacian regime" it behaves more or less like a symmetric differentiation operator. For higher orders, the filter's frequency response gets more and more concentrated at the high frequencies as it enters the "Gabor regime", where it behaves directional.

The presented formulas only provide the filters at one side of the wavelet transform (resp. analysis/synthesis). The filters corresponding to the other side can be found as their dual. This leads to the expressions of Table 1. Of course, the filters can be interchanged between the analysis and synthesis side, depending on the application.

\section{RESULTS}

\subsection{Implementation}

None of the filters listed in Table 1 is compactly supported. However, we have implemented the wavelet transform using the Fourier transform as in $[7,8]$. Therefore, there is no need for compactly supported filters and periodic boundary conditions are automatically implied. Also note that all filters are known analytically in the Fourier domain. Another important implementation issue is the calculation of the autocorrelation filter $A\left(e^{j \omega}\right)$, which is obtained in the Fourier domain as well via an iterative numerical procedure, that we have developped recently [9].

\footnotetext{
filter.
} 
Table 1. Overview of isotropic-polyharmonic B-spline wavelet transform

\begin{tabular}{|l|l|}
\hline \hline \multicolumn{2}{|c|}{ analysis side } \\
\hline scaling filter & $\begin{array}{l}\tilde{H}(\mathbf{z})=\frac{A(\mathbf{z})}{A\left(\mathbf{z}^{\mathrm{D}}\right)} B_{\gamma}(\mathbf{z}) \\
\tilde{G}(\mathbf{z})=-z_{1}^{-1} \frac{A(\mathbf{z}) B_{\gamma}\left(-\mathbf{z}^{-1}\right)}{A\left(\mathbf{z}^{\mathrm{D}}\right) A(-\mathbf{z})}\end{array}$ \\
\hline wavelet filter & \multicolumn{2}{|c|}{ synthesis side } \\
\hline \hline scaling filter & $H(\mathbf{z})=B_{\gamma}(\mathbf{z})$ \\
wavelet filter & $G(\mathbf{z})=-z_{1}^{-1} B_{\gamma}\left(-\mathbf{z}^{-1}\right) A(-\mathbf{z})$ \\
\hline \hline
\end{tabular}

(a)

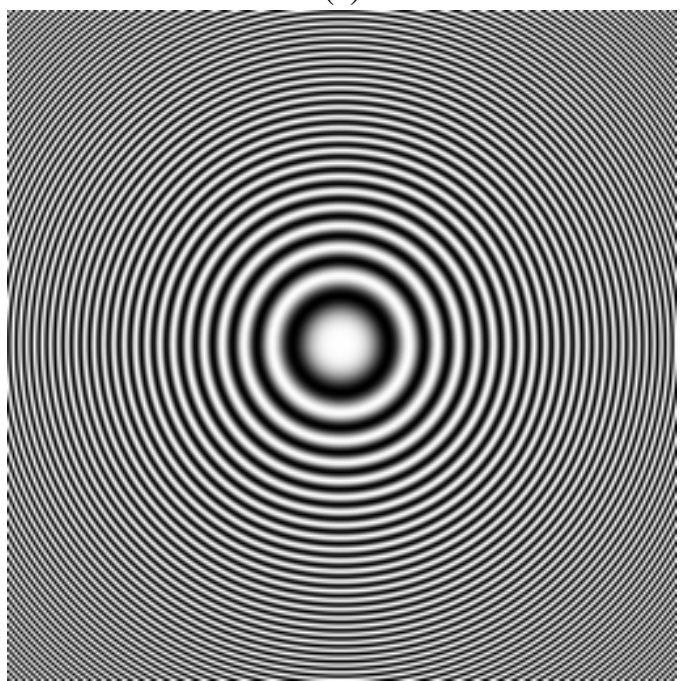

(b)

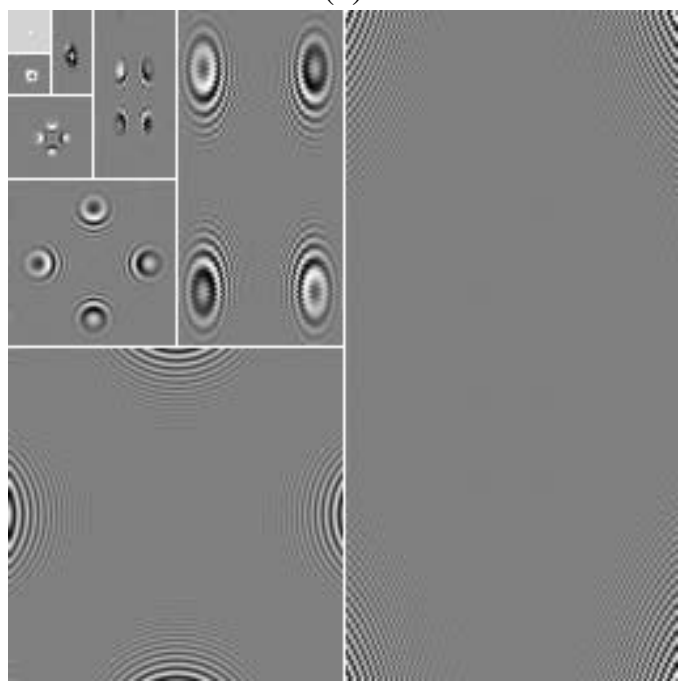

Fig. 3. (a) Test image 'zoneplate'. (b) Decomposition of 'zoneplate' for 8 iterations and order $\gamma=5$.

\subsection{Some examples}

In Fig. 3 we show an example wavelet decomposition of the "zoneplate" image. The subbands are organized in a way that is standard for the quincunx subsampling scheme; i.e., for odd iterations the odd lines are shifted by one pixel and then odd columns are subsampled. The intensity values within each subband of Fig. 3 (b) have been rescaled to improve visualization.

In the first subband, we only capture high frequency components at the corners of the frequency sweep. In fact, the local spatial frequency at these corners is close to the center frequency of the corresponding Gabor atoms depicted in Fig. 2 (b). As we progress through scale, the "sensitive" regions detected by the wavelet have lower spatial frequencies. Interestingly, at some juncture, we switch from "Gabor regime" to "Laplacian regime".

\section{CONCLUSIONS \& OUTLOOK}

We proposed a new wavelet transform based on the isotropic polyharmonic B-splines. These wavelets were designed to be semi-orthogonal.

While due to space constraints, we limited ourselves to the description of one particular wavelet-the B-spline one-, there are other interesting members in the family, including the more 'classical' orthonormal one.

\section{ACKNOWLEDGEMENTS}

This work is funded in part by the grant 200020-101821 from the Swiss National Science Foundation. Brigitte Forster also acknowledges the financial support from the German Academy of Natural Scientists Leopoldina (BMBF-LPD 9901/8-64) and from the European Union's Human Potential Program (HPRN-CT-2002-00285).

\section{REFERENCES}

[1] C. Rabut, "Elementary $m$-harmonic cardinal B-splines," Numerical Algorithms, vol. 2, pp. 39-62, 1992.

[2] W. R. Madych, Wavelets: A tutorial in theory and applications, chapter Some elementary properties of multiresolution analyses of $L_{2}\left(\mathbb{R}^{n}\right)$, pp. 259-294, Academic Press, Boston, 1992.

[3] D. Van De Ville, T. Blu, and M. Unser, "Isotropic polyharmonic Bsplines: Scaling functions and wavelets," IEEE Transactions on Image Processing, to appear.

[4] M. Vetterli, "Multi-dimensional sub-band coding: Some theory and algorithms," Signal Processing, vol. 6, no. 2, pp. 97-112, Feb. 1984.

[5] J.-C. Feauveau, Analyse multirésolution par ondelettes non orthogonales et bancs de filtres numériques, Ph.D. thesis, Univ. Paris Sud, 1990.

[6] M. Unser, A. Aldroubi, and M. Eden, "A family of polynomial spline wavelet transforms," Signal Processing, vol. 30, pp. 141-162, 1993.

[7] F. Nicolier, O. Laligant, and F. Truchetet, "Discrete wavelet transform implementation in Fourier domain for multidimensional signal," Journal of Electronic Imaging, vol. 11, no. 3, pp. 338-346, July 2002.

[8] M. Feilner, D. Van De Ville, and M. Unser, "An orthogonal family of quincunx wavelets with continuously-adjustable order," IEEE Transactions on Image Processing, to appear.

[9] T. Blu, D. Van De Ville, and M. Unser, "Numerical methods for the computation of wavelet correlation sequences," SIAM Journal on $\mathrm{Nu}$ merical Analysis, submitted. 